\title{
Use of dynamic movement orthoses to improve gait stability and trunk control in ataxic patients
}

\author{
Mariano SERRAO 1,2 *, Carlo CASALI ${ }^{1}$, Alberto RANAVOLO ${ }^{3}$, Silvia MARI ${ }^{4}$, Carmela CONTE 4 , \\ Giorgia CHINI 2, 3, Luca LEONARDI 1, Gianluca COPPOLA 5, Cherubino DI LORENZO 1, \\ Mahmoud HARFOUSH ${ }^{2}$, Luca PADUA 4, 6, Francesco PIERELLI 1,7
}

\begin{abstract}
${ }^{1}$ Department of Medical and Surgical Sciences and Biotechnologies, Sapienza University, Polo Pontino, Latina, Italy; ${ }^{2}$ Movement Analysis Lab, Rehabilitation Center, Policlinico Italia Private Clinic, Rome, Italy; ${ }^{3}$ Department of Occupational and Environmental Medicine, Epidemiology, and Hygiene, INAIL, Rome, Italy; ${ }^{4}$ Don Gnocchi Foundation, Milan, Italy; ${ }^{5}$ Department of Neurophysiology of Vision and Neuro-ophthalmology, G.B. Bietti Foundation and Institute for Research and Care, Rome, Italy; ${ }^{6}$ Department of Geriatrics, Neuroscience and Orthopedics, Sacro Cuore Catholic University, Rome, Italy; ${ }^{7}$ Neuromed Institute for Research and Care, Pozzilli, Italy

*Corresponding author: Mariano Serrao, Department of Medical and Surgical Sciences and Biotechnologies, Sapienza University, Polo Pontino, Latina, Italy. E-mail: mariano.serrao@uniroma1.it
\end{abstract}

\section{A B S T R A C T}

BACKGROUND: Patients with cerebellar ataxia show increased upper body movements, which have an impact on balance and walking. AIM: In this study, we investigated the effect of using dynamic movement orthoses (DMO), designed as elastic suits, on trunk motion and gait parameters.

DESIGN: Longitudinal uncontrolled study.

SETTING: Outpatient rehabilitation unit.

POPULATION: Eleven patients ( 7 men, 4 women; mean age: $49.9 \pm 9.5$ years) with degenerative cerebellar ataxia were enrolled in this study. METHODS: Linear overground gait of patients was recorded by means of an optoelectronic gait analysis system before DMO use (DMO-) and during $\mathrm{DMO}$ use (DMO+). Time-distance parameters, lower limb joint kinematics, body sway, trunk oscillations, and gait variability (coefficient of variation [CV]) were recorded. Patient satisfaction with DMO device was measured using Quebec user evaluation of satisfaction with assistive technology.

RESULTS: When using the DMO, patients showed a significant decrease in stance phase duration, double support phase duration, swing phase $\mathrm{CV}$, pelvic range of motion (ROM), body sway, and trunk ROMs. A significant increase was observed in the swing phase duration and knee joint ROM. Out of 11 patients, 10 were either quite satisfied ( 8 points) or very satisfied ( 2 points) with the assistive device.

CONCLUSIONS: The DMO reduce the upper body motion and in improve balance-related gait parameters.

CLINICAL REHABILITATION IMPACT: We propose use of DMO as an assistive/rehabilitative device in the neurorehabilitation of cerebellar ataxia to improve the trunk control and gait stability. DMO may be considered a prototype that can be modified in terms of material characteristics, textile layers, elastic components, and diagonal and lateral seams.

(Cite this article as: Serrao M, Casali C, Ranavolo A, Mari S, Conte C, Chini G, et al. Use of dynamic movement orthoses to improve gait stability and trunk control in ataxic patients. Eur J Phys Rehabil Med 2017;53:735-43. DOI: 10.23736/S1973-9087.17.04480-X)

Key words: Orthotic devices - Rehabilitation - Walking - Postural balance - Gait ataxia.

$\mathrm{P}$ atients with cerebellar ataxia typically exhibit clumsy, staggering movements with a wide-based gait. ${ }^{1}$ One of the main features of gait ataxia is increased variability of all global and segmental gait parameters, ${ }^{2}$ which is an alteration known to be linked with an in- creased risk of fall. ${ }^{3}$ Recently, upper body oscillations, which had long been reported in literature as a clinical feature of ataxia, ${ }^{4}$ have been quantitatively characterized in ataxic patients. ${ }^{5,6}$ Wide upper body oscillations that move the center of mass toward the edges of the 


\section{COPYRIGHT $^{(} 2017$ EDIZIONI MINERVA MEDICA}

SERRAO

DYNAMIC MOVEMENT ORTHOSES IN ATAXIC PATIENTS

TABLE I.-Patients' characteristics.

\begin{tabular}{|c|c|c|c|c|c|c|c|c|}
\hline \multirow{2}{*}{ Patient \# } & \multirow{2}{*}{ Diagnosis } & \multirow{2}{*}{$\underset{\text { (years) }}{\text { Age }}$} & \multirow{2}{*}{$\begin{array}{c}\text { Age at onset } \\
\text { (years) }\end{array}$} & \multirow{2}{*}{$\begin{array}{l}\text { Duration } \\
\text { (months) }\end{array}$} & \multicolumn{4}{|c|}{ ICARS scores } \\
\hline & & & & & Gait & Posture & Lower limb kinetics & Total \\
\hline 1 & SAOA & 59 & 47 & 12 & 3 & 4 & 4 & 12 \\
\hline 2 & SAOA & 60 & 50 & 10 & $7(6)$ & 11 & 14 & $42(41)$ \\
\hline 3 & SAOA & 48 & 30 & 18 & 4 & 9 & 7 & 26 \\
\hline 4 & SCA1 & 57 & 45 & 12 & 9 & $15(14)$ & 4 & $36(35)$ \\
\hline 5 & SCA1 & 44 & 35 & 9 & 1 & 1 & 1 & 6 \\
\hline 6 & SCA2 & 38 & 30 & 8 & 4 & 10 & 8 & 49 \\
\hline 7 & SCA1 & 50 & 41 & 9 & 4 & 5 & 2 & 18 \\
\hline 8 & SAOA & 73 & 60 & 13 & 3 & 3 & 1 & 11 \\
\hline 9 & SAOA & 49 & 17 & 32 & 4 & 5 & 2 & 18 \\
\hline 10 & SAOA & 50 & 30 & 20 & 1 & 0 & 1 & 6 \\
\hline 11 & SAOA & 56 & 40 & 8 & 5 & 10 & 8 & 30 \\
\hline
\end{tabular}

Values in parentheses indicate ICARS scores at $\mathrm{T} 1$ follow-up.

ICARS: International Cooperative Ataxia Rating Scale; SAOA: sporadic adult onset ataxia; SCA: spinocerebellar ataxia.

base of support may worsen gait instability, increase body sway during walking, and further increase the risk of fall. It has been recently shown that ataxic patients adopt a strategy to control their walking instability that involves an increase in antagonistic muscle co-activation and widening of muscle activation timing to stiffen the body segments. 6,7 However, this compensatory mechanism has some negative effects, such as increased metabolic cost and risk of cartilage degeneration. . $^{8-10}$ Based on the aforementioned gait abnormalities, ataxic patients need to use specific devices aimed at stabilizing the upper body, minimizing body sway during walking and reducing walking variability. Theoretically, ataxic patients may benefit from using elastic or semi-rigid orthoses that can reduce trunk oscillations and stabilize joint trajectories without restricting lower limb movements during walking.

Dynamic Lycra movement orthoses (DMO) have been used in recent years as a treatment tool for children with motor and posture impairment, such as scoliosis and cerebral palsy. ${ }^{11-17}$ These elastic orthoses can be designed as suits with a snug fit. The resistance created by the inherent properties of Lycra fabric, the additional layers of reinforcing material as well as the diagonal and lateral seams from the shoulder to the pelvis add a biomechanical influence to constrain or stabilize body segments of the trunk and hip joints on all spatial planes. Furthermore, increased pressure on certain muscle groups may improve proprioception and may facilitate joint movements.
This study primarily aimed to investigate the effect of DMO use, designed as elastic suits, on time-distance gait parameters, motion of lower limb joints, body sway, trunk oscillations, and gait variability in patients with degenerative primary cerebellar ataxia. Second, the study aimed to evaluate the patient satisfaction with these elastic devices.

\section{Materials and methods}

Eleven patients ( 7 men, 4 women; mean age: $49.9 \pm 9.5$ years) with degenerative cerebellar ataxia were enrolled in this study. Four were diagnosed with autosomal dominant ataxia (spinocerebellar ataxia [SCA]; three patients with SCA1 and one patients with SCA2) while the other seven had sporadic adult-onset ataxia of unknown etiology.

Since patients with SCA may have impairment of other systems in addition to cerebellar symptoms, we only included those patients whose gait disturbance was exclusively cerebellar in nature. Accordingly, we excluded patients with major involvement of neurological systems (e.g., extrapyramidal, pyramidal, peripheral nerve or muscle) other than cerebellar impairment as well as those with orthopedic disorders that could cause further gait impairment, such as severe scoliosis, painful musculoskeletal conditions, and foot deformities. Patients did not have visual impairment (e.g., optic atrophy or retinitis pigmentosa) but almost all had oculomotor abnormalities such as 


\section{COPYRIGHT $^{(} 2017$ EDIZIONI MINERVA MEDICA}

DYNAMIC MOVEMENT ORTHOSES IN ATAXIC PATIENTS

SERRAO

gaze nystagmus or square-wave jerks during pursuit movements. All patients showed cerebellar atrophy on magnetic resonance imaging and could walk without assistance or walking aids along a 10-meter laboratory walkway. Patients' characteristics at the time of the enrollment are summarized in Table I. Disease severity was rated using the International Cooperative Ataxia Rating Scale (ICARS). ${ }^{18}$ None of the enrolled patients received rehabilitative treatment. All of them were instructed to perform common daily activities when wearing the DMO. All the participants gave a written informed consent according to the Declaration of Helsinki. The local research ethics committee approved the study.

\section{$D M O s$}

DMOs are made of extensible material (Lycra) that was stitched together. Each panel or section is made using specific tensions and directions of pull to support the patient's need for maintaining biomechanical alignment. 16, 17 Extra layering can be provided to further reduce movements of body segments in specific directions. For this study, elastic suits (Figure 1) were custom designed for each patient according to his/her anthropometric measurements. The DMO suit extended from the shoulders (above the elbow joints) to the hips (above the knee joints). The DMO was measured to provide a snug fit and to support the shoulder and hip girdles. The suit that connected the shoulders and the pelvis created a low force that resisted trunk movements. Diagonal and lateral seams from the shoulder to the pelvis were added to further increase resistance to trunk movements in the sagittal and lateral directions (Figure 1). For each patient, three extra layers were added to reinforce the inherent properties of Lycra in creating low forces resistant to trunk movement. When necessary, zips were present but were kept to a minimum to preserve traction. Lycra DMO usually took 2-5 minutes to put on. It had appropriate apertures for toileting.

\section{Gait analysis}

An optoelectronic motion analysis system (SMARTDX 500 System, BTS Bioegineering Italia, Garbagnate Milanese, Milan, Italy) consisting of eight infrared cam-

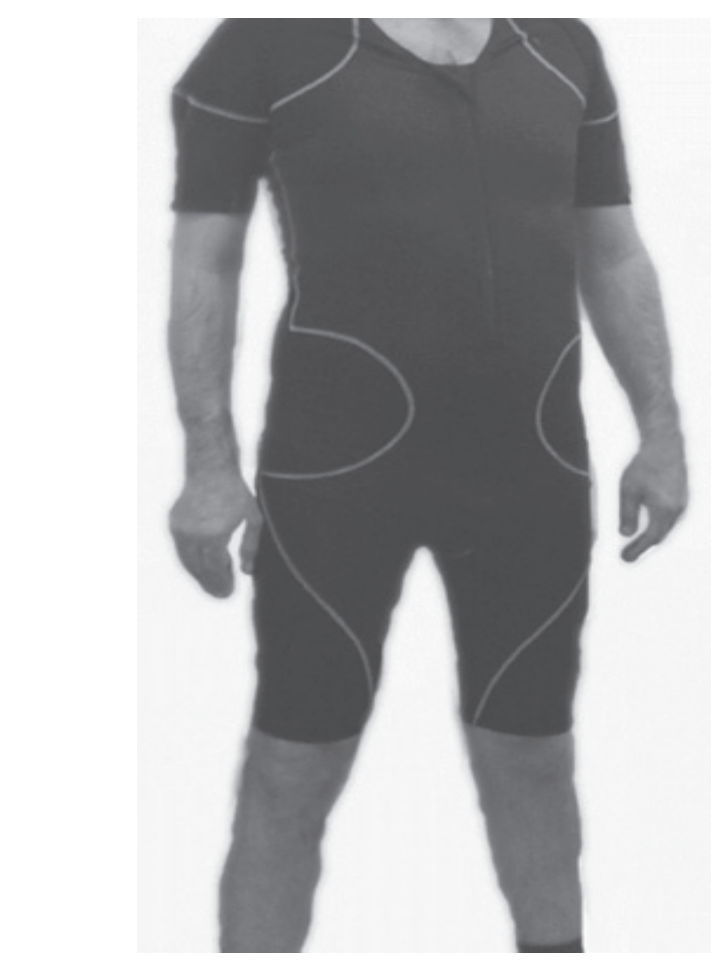

Figure 1.-Example of individually custom designed elastic suit with three layers of Lycra tissue in a patient with cerebellar ataxia.

eras with sampling frequency of $300 \mathrm{~Hz}$ was used to detect the movements of 20 reflective spherical markers placed over anatomical landmarks according to Davis et al. ${ }^{19}$ Two heel markers were added for standing trials (off-set angle calculation). Anthropometric data were collected for each subject. ${ }^{20}$ Participants were required to walk barefoot at self-selected speed along a 10-meter walkway.

\section{Patient satisfaction evaluation}

Patient satisfaction with the DMO device and service was measured using Quebec User Evaluation of Satisfaction with Assistive Technology (QUEST).21, 22 The QUEST survey contains 15 items categorized into two sub-scales with which patients rate the assistive device and the service they received. Six questions were used to rate patient satisfaction using a 5-point scale ranging from "very dissatisfied" to "very satisfied." The 5-point patient satisfaction scale was split into two categories. Scores of 1 and 2 belong to one category and scores of 3,4 , and 5 belong to the other. 


\section{COPYRIGHT $^{(} 2017$ EDIZIONI MINERVA MEDICA}

SERRAO

DYNAMIC MOVEMENT ORTHOSES IN ATAXIC PATIENTS

\section{Procedure}

Two gait analysis sessions were performed for each patient. The first session was performed before they used the DMO (DMO-) and a second session was performed one month after using the Lycra suit for 2-6 hours a day and 3-5 days a week. In the second session, gait analysis was performed while wearing the $\mathrm{DMO}(\mathrm{DMO}+) . \mathrm{Pa}-$ tients were then required to fill in the QUEST questionnaires. Furthermore, they were also asked about actual compliance in wearing the device.

\section{Data analysis}

Three-dimensional marker trajectories were acquired using a frame-by-frame acquisition and tracking software (SMART Capture and SMART Tracker, BTS Bioengineering). Data were processed using a 3D reconstruction software (SMART Analyzer, BTS Bioengineering). Kinematic data were normalized between two consecutive heel strikes reduced to 100 samples over the gait cycle using a polynomial procedure. Data were analyzed with the right and left limb considered together.

\section{Time-distance parameters}

The following time-distance parameters were considered for the statistical analysis: step length (meters) and width (meters), stance duration (\%), swing duration $(\%)$, and double support duration (\%).

\section{Lower limb joint kinematics}

Mean range of motion (ROM) values of the hip, knee, and ankle joints were calculated in the sagittal plane. Mean ROM values of the pelvis were also calculated in the sagittal (tilt movements), frontal (obliquity movements), and transverse (rotation movements) planes.

\section{Body sway and trunk oscillations}

We calculated the maximal linear displacement of the center of the pelvis in the mediolateral direction to measure whole body sway during walking. We measured the ROM of the trunk segment in the sagittal, frontal, and transverse planes to assess upper body motion during walking. In particular, trunk movement measure- ments were obtained using an indirect method based on shoulder girdle landmarks. $5,23,24$ With regards to the trunk trajectories in the sagittal and frontal planes, we calculated the centroid of the triangle formed by acromion markers and $\mathrm{C} 7$. We also calculated the centroid of the triangle formed by the anterior superior iliac spine (ASIS) and sacral markers (pelvis level, P). The trunk $(\mathrm{T})$ coordinate system, which is a system embedded with the shoulder segment, has a vertical axis (V) aligned with the gravity vector, a mediolateral axis (ML) passing from the right acromion to the left acromion and an anterior posterior axis (AP) computed as the cross product of the ML and V vectors. The pelvis coordinate system, which is a system embedded with the pelvis segment, has a vertical axis aligned with the gravity vector, the mediolateral axis (ML) passing from the right ASIS to the left ASIS and the anterior posterior axis (AP) computed as the cross product of the ML and $\mathrm{V}$ vectors. The trunk functions $\left(f_{\text {Trunk }}\right)$ were calculated as the differences, measured in meters, between the trunk and pelvis trajectories, respectively.

\section{Variability measures}

As an index of within-subject variability, we calculated the coefficient of variation $(\mathrm{CV})$ for the time-distance and lower limb joint kinematic variables according to the following formula:

$$
\mathrm{CV}=\frac{\sigma}{|\mu|}
$$

where $\sigma$ indicates the standard deviation and $\mu$ the mean value of the considered parameter.

\section{Statistical analysis}

Either paired $t$-test or non-parametric Wilcoxon signed-rank test for paired samples was used to investigate differences between the two $\mathrm{DMO}+$ and $\mathrm{DMO}-$ sessions in the analyzed variables according to the normal or non-normal data distribution. Chi-square/Fisher tests were used to evaluate differences in patients' questionnaire scores in both $\mathrm{DMO}-$ and $\mathrm{DMO}+$ sessions relative to questionnaire outcomes. Data were presented as mean \pm standard error. $\mathrm{P}$ value $<0.05$ was considered statistically significant. 


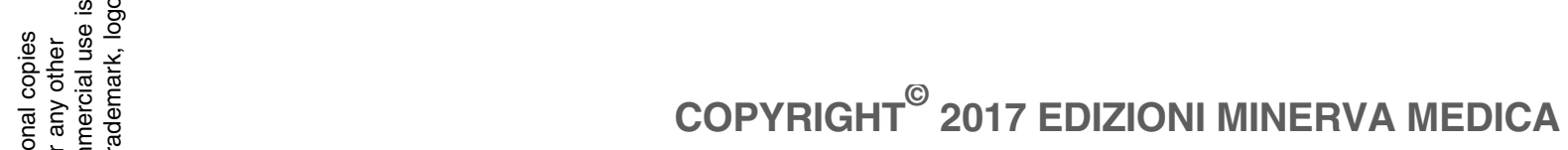

DYNAMIC MOVEMENT ORTHOSES IN ATAXIC PATIENTS

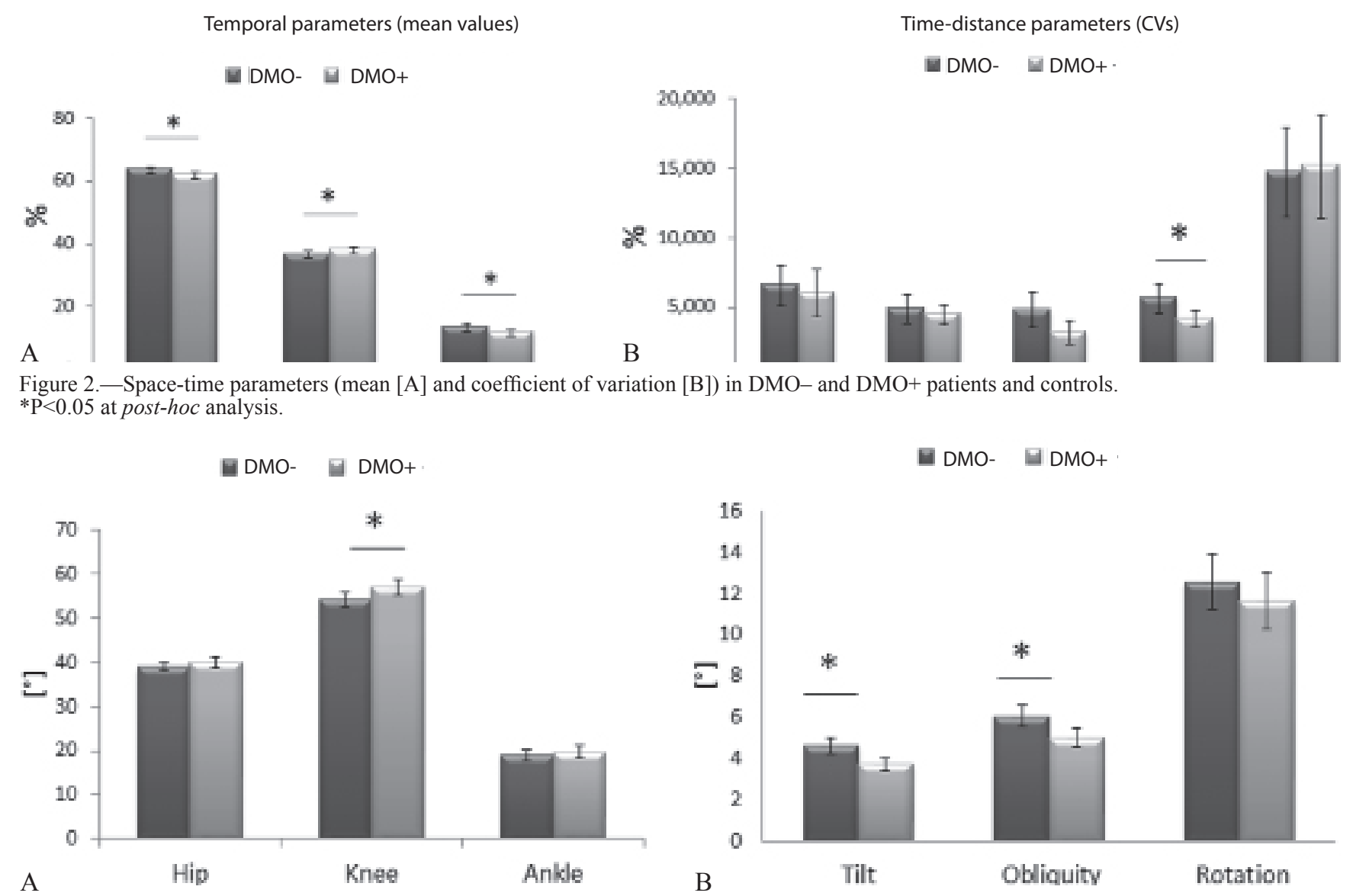

Figure 3.-Hip, knee, and ankle joint ROM in the sagittal plane (A) and pelvic ROM (B) on the sagittal (tilt movements), frontal (obliquity movements), and transverse (rotation movements) planes.

$* \mathrm{P}<0.05$ at post-hoc analysis.

\section{Results}

\section{Time-distance parameters}

A significant effect of $\mathrm{DMO}+/-$ sessions was found on stance (main effect, $F_{(2,20)}=4.726, \mathrm{P}=0.021$ ) and double support phase (main effect: $F_{(2,20)}=6.800, \mathrm{P}=0.006$ ) durations. No significant differences were found for step length, step width, and speed mean values $(\mathrm{P}>0.05)$. Post-hoc pairwise comparisons showed lower stance duration values in post-DMO+ session than pre-DMO- session and lower double support duration in post-DMO+ than both in pre-DMO+ and post-DMO- (Figure 2).

Significant lower $\mathrm{CV}$ values were found for swing phase duration in $\mathrm{DMO}+$ session than in $\mathrm{DMO}$ - (Figure 2). No significant differences were found for CV values of the other time-distance variables $(\mathrm{P}>0.05)$.

\section{Lower limb joint kinematics}

Significantly higher mean ROM values of the knee joint were found in $\mathrm{DMO}+$ session than in the DMOsession (Figure 3). No significant differences were found for both mean ROM values of the hip and ankle joints and for CV values of all lower limb joints $(\mathrm{P}>0.05)$.

A significant decrease in the mean ROM values of the pelvis were found in $\mathrm{DMO}+$ compared to that in DMO - in both the sagittal and frontal planes (Figure 3), while no differences were found on the transverse plane $(\mathrm{P}>0.05)$. There was also no difference in $\mathrm{CV}$ values $(\mathrm{P}>0.05)$.

Power analysis results are displayed in Supplementary Table I (online content only). 



\section{COPYRIGHT $^{(} 2017$ EDIZIONI MINERVA MEDICA}

one category while scores of 3,4 , and 5 belonged to the other one.

Ten out of 11 patients $(90.9 \%)$ were either quite satisfied ( 8 points) or very satisfied ( 2 points) with the assistive device while only one patient was somewhat satisfied $\left(\mathrm{P}<0.001\right.$ by $\chi^{2}$ test $)$.

Ten out of 11 patients $(81.8 \%)$ were also either quite satisfied (1 patient) or very satisfied ( 8 patients) with the device while two patients $(18.1 \%)$ were not very satisfied (1 patient) or were more or less satisfied (1 patient) $\left(\mathrm{P}<0.001\right.$ by $\chi^{2}$ test $)$.

Patients reported wearing the DMO for an average of 4.7 \pm 1.1 hours per day (range: $3-6$ hours) for $4.5 \pm 0.5$ times per week (range 4-5).

\section{Discussion}

In this study, we investigated the effect of DMO use, designed as wearable suits, on the space-time parameters, joint and pelvis ROMs, gait variability, body sway, and trunk oscillations in patients with degenerative cerebellar ataxia.

We found that DMO induced the following: 1) a decrease in stance and double support phase durations, an increase in swing phase duration, and a decrease in swing duration variability; 2) an increase in knee joint ROMs in the sagittal plane and a decrease in pelvis ROMs in both the sagittal and frontal planes; and 3) a decrease in body sway in the mediolateral direction and a decrease in trunk oscillations on sagittal plane. Furthermore, patients subjectively had a high level of satisfaction with the Lycra elastic suit and the resulting outcomes.

Various time-distance parameter abnormalities have been described in ataxic patients, including increased stance and double support phase durations and step width as well as reduced step length and gait speed.2, 25-28 Most of these abnormalities seemed to represent compensatory mechanisms aimed at reducing dynamic imbalance. In our study, reduced stance and double support phase durations as well as increased swing phase duration observed during the $\mathrm{DMO}+$ session indicated that patients felt safer wearing the elastic suit. Thus, patients had lesser need to walk in bipodalic stance, which is a more stable configuration compared to monopodalic stance.

With regard to joint kinematics, we found that DMO reduced the pelvic ROM in both the frontal and sagittal planes. Our findings are consistent with previous findings on the effect of lower limb Lycra garments on the gait of children with cerebral palsy and muscular dystrophy 14 and suggest that one of the most relevant effects of the DMO was to stabilize the pelvis during walking without restraining movement of the hip, knee, and ankle joints. Unexpectedly, we found an increase in knee ROMs during DMO+. This may be a consequence of improved limb stabilization during swing phase, which was also revealed by reduced swing phase duration variability. Thus, it may reflect a better control of knee flexion during swing phase.

It had been shown that patients with cerebellar ataxia showed an irregular gait pattern with large body sway in the mediolateral direction. ${ }^{2,} 6$ Furthermore, ataxic patients presented large oscillations of their upper body. 5 Such abnormalities may be attributed to impaired multi-joint and multi-segment coordination as well as to muscle hypotonia. $1,29,30$ Wide oscillations of the upper body may greatly affect gait performance and gait stability. ${ }^{5}$ Thus, reducing body sway and trunk oscillations may be an important aspect in the rehabilitation of these patients. Indeed, it is known that minimizing the magnitude of linear and angular displacement of the head and trunk had functional importance in ensuring clear vision, ${ }^{31,32}$ facilitating the integration of vestibular information, ${ }^{33}$ and contributing to the maintenance of balance. ${ }^{34,} 35$ Our study showed that the Lycra elastic DMO succeeded in reducing trunk oscillations in the sagittal plane, body sway in the mediolateral direction, as well as the pelvic ROM in both the sagittal and frontal planes. All these effects were possibly due to reinforcement in the functional connection between the shoulders and pelvis provided by the DMO. Using the diagonal and lateral seams with additional layers may have contributed in increasing resistance to movements of the upper body and pelvis, which then reduced body sway during walking.

Another important feature of patients with ataxia is gait variability, which is an indirect sign of dynamic instability of locomotion and is associated with increased risk of falls. ${ }^{3}, 36,37$ In this study, we found that DMO use specifically reduced the variability of swing phase duration. In our previous study, ${ }^{5}$ we showed that the increased upper body oscillations were associated with increased swing phase variability. This suggested 


\section{COPYRIGHT $^{(} 2017$ EDIZIONI MINERVA MEDICA}

SERRAO

that exaggerated upper body movements may influence some specific aspects of gait instability, mainly those related to the timing before foot placement and to the maintenance of a regular temporal pattern during walking. ${ }^{5}$ In light of this finding, reduced trunk oscillations caused by DMO may indirectly improve stability of the swinging limb.

\section{Limitations of the study}

In our study, all patients reported to be more stable and safer in performing daily life activities when wearing the DMO. Since patients had no rehabilitative treatment during the one-month trial period, the observed gait parameter improvements may be exclusively attributed to the use of DMO.

We focused our study mostly on the direct biomechanical effect of the DMO in reducing the upper body motion and in improving balance-related gait parameters (e.g., stance and double support phase durations and gait variability). Patients were asked to wear the DMO for about one month to allow them to adapt to the device, to train, and to assess their perception in using it. However, since, after one month of DMO use, we did not investigate the patients also without DMO we could not discern the rehabilitative effect from the assistive effect. Furthermore, we did not compare the acute effect and one-month use effect. Other rehabilitative methods focused on balance and postural disorders rehabilitation, such as torso-weighting, revealed immediate advantages over a non-weighted condition for static standing and gait in patients with ataxia and multiple sclerosis. ${ }^{38-40}$ Further studies with long-term are warranted to understand how and what extend the central nervous system adapt to the chronic use of the DMO.

Other limitations of this study include the potential for placebo (sham) effect and the small sample size. The lack of a placebo-control condition cannot exclude a bias caused by placebo-induced amelioration. However, to our knowledge, there is no evidence in the literature of a placebo effect on kinematic measurements. Although our sample size was small, this limitation was partly offset by the adoption of sensitive quantitative measures of motion. Furthermore, in our study we enrolled patients with different degrees of disease severity (ICARS scores ranging from 6 to 49), even though all of them were able to walk without assistance for at least few steps. The small sample size did not allow us to sub-grouping patients in order to evaluate if the use of the DMO was more effective in mild or in severe/ moderate ataxia. Nevertheless, since our study was hypothesis-generating in nature, a larger study would be needed to confirm the findings presented here and to better evaluate the DMO effect in patients with different disease severity.

\section{Conclusions}

In conclusion, we propose the use of DMO in the neurorehabilitation of cerebellar ataxias to improve trunk control and gait stability. However, the DMO may be considered a prototype that can be modified in terms of material characteristics, textile layers, elastic components, and diagonal and lateral seams to improve gait stability in ataxic patients. Further studies are needed to evaluate its long-term effects.

\section{References}

1. Bodranghien F, Bastian A, Casali C, Hallett M, Louis ED, Manto M, et al. Consensus Paper: Revisiting the Symptoms and Signs of Cerebellar Syndrome. Cerebellum 2016;15:369-91.

2. Serrao M, Pierelli F, Ranavolo A, Draicchio F, Conte C, Don R, et al. Gait pattern in inherited cerebellar ataxias. Cerebellum 2012;11:194211.

3. Schniepp R, Wuehr M, Schlick C, Huth S, Pradhan C, Dieterich M, et $a l$. Increased gait variability is associated with the history of falls in patients with cerebellar ataxia. J Neurol 2014;261:213-23.

4. Holmes G. The cerebellum of man. Brain 1939:62:1-30.

5. Conte C, Pierelli F, Casali C, Ranavolo A, Draicchio F, Martino G, et al. Upper body kinematics in patients with cerebellar ataxia. Cerebellum 2014;13:689-97.

6. Martino G, Ivanenko YP, Serrao M, Ranavolo A, d'Avella A, Draicchio F, et al. Locomotor patterns in cerebellar ataxia. J Neurophysiol 2014;112:2810-21.

7. Mari S, Serrao M, Casali C, Conte C, Martino G, Ranavolo A, et al. Lower limb antagonist muscle co-activation and its relationship with gait parameters in cerebellar ataxia. Cerebellum 2014;13:226-36.

8. Peterson DS, Martin PE. Effects of age and walking speed on coactivation and cost of walking in healthy adults. Gait Posture 2010;31:355-9.

9. Falconer K, Winter DA. Quantitative assessment of co-contraction at the ankle joint in walking. Electromyogr Clin Neurophysiol $1985 ; 25: 135-49$

10. Griffin TM, Guilak F. The role of mechanical loading in the onset and progression of osteoarthritis. Exercise Sport Sci Rev 2005;33:195200.

11. Elliott C, Reid S, Hamer P, Alderson J, Elliott B. Lycra( $\left({ }^{\circledR}\right)$ arm splints improve movement fluency in children with cerebral palsy. Gait Posture 2011:33:214-9.

12. Matthews M, Crawford R. The use of dynamic Lycra orthosis in the treatment of scoliosis: a case study. Prosthet Orthot Int 2006:30:174-81

13. Watson MJ, Crosby P, Matthews M. An evaluation of the effects of a dynamic lycra orthosis on arm function in a late stage patient with acquired brain injury. Brain Inj 2007;21:753-61. 


\section{COPYRIGHT $^{(} 2017$ EDIZIONI MINERVA MEDICA}

DYNAMIC MOVEMENT ORTHOSES IN ATAXIC PATIENTS

SERRAO

14. Rennie DJ, Attfield SF, Morton RE, Polak FJ, Nicholson J. An evaluation of lycra garments in the lower limb using 3-D gait analysis and functional assessment (PEDI). Gait Posture 2000;12:1-6.

15. Hylton N, Allen C. The development and use of SPIO Lycra compression bracing in children with neuromotor deficits. Pediatr Rehabil 1997;1:109-16.

16. Corn K, Imms C, Timewell G, Carter C, Collins L, Dubbeld S, et al. Impact of second skin lycra splinting on the quality of upper limb movement in children. Br J Occup Ther 2003;66:464-72.

17. Wilton JC. Hand splinting: principles of design and fabrication. London: W.B. Saunders; 1997. p.168-97.

18. Trouillas P, Takayanagi T, Hallet M, Currier RD, Subramony SH, Wessel K, et al. International Cooperative Ataxia Rating Scale for pharmacological assessment of the cerebellar syndrome. The Ataxia Neuropharmacology Committee of the World Federation of Neurology. J Neurol Sci 1997;145:205-11.

19. Davis RB 3rd, Õunpuu S, Tyburski D, Gage JR. A gait analysis data collection and reduction technique. Hum Mov Sci 1991;10:575-87.

20. Winter DA. Biomechanics of human movement. New York: Wiley; 1979.

21. Demers L, Weiss-Lambrou R, Ska B. Development of the Quebec user evaluation of satisfaction with assistive technology (QUEST). Assist Technol 1996;8:3-13.

22. Demers L, Weiss-Lambrou R, Ska B. Item analysis of the Quebec User Evaluation of Satisfaction with Assistive Technology (QUEST). Assist Technol 2000;12:96-105.

23. Cappozzo A, Figura F, Leo T, Marchetti M. Movements and mechanical energy changes in the upper part of the body during walking. In: Assmussen E, Jorgansen K, editors. Biomechanics VI-A. Baltimore: University Park Press; 1978. p. 272-9.

24. Krebs DE, Wong D, Jevsevar D, Riley PO, Hodge WA. Trunk kinematics during locomotor activities. Phys Ther 1992;72:505-14.

25. Palliyath S, Hallett M, Thomas SL, Lebiedowska MK. Gait in patients with cerebellar ataxia. Mov Disord 1998;13:958-64.

26. Ebersbach G, Sojer M, Valldeoriola F, Wissel J, Müller J, Tolosa E, et al. Comparative analysis of gait in Parkinson's disease, cerebellar ataxia and subcortical arteriosclerotic encephalopathy. Brain 1999;122:1349-55

27. Mitoma H, Hayashi R, Yanagisawa N, Tsukagoshi H. Characteristics of parkinsonian and ataxic gaits: a study using surface electromyograms, angular displacements and floor reaction forces. J Neurol Sci 2000;174:22-39.

28. Stolze H, Klebe S, Petersen G, Raethjen J, Wenzelburger R, Witt K, et al. Typical features of cerebellar ataxic gait. J Neurol Neurosurg Psychiatry 2002;73:310-2.

29. Thach WT, Goodkin HP, Keating JG. The cerebellum and the adaptive coordination of movement. Annu Rev Neurosci 1992;15:403-42.

30. Salman ME. The cerebellum: it's about time! But time is not everything-new insights into the role of the cerebellum in timing motor and cognitive tasks. J Child Neurol 2002;17:1-9.

31. Grossman GE, Leigh RJ, Abel LA, Lanska DJ, Thurston SE. Frequency and velocity of rotational head perturbations during locomotion. Exp Brain Res 1989;70:470-6.

32. Hirasaki E, Moore ST, Raphan T, Cohen B. Effects of walking velocity on vertical head and body movements during locomotion. Exp Brain Res 1999;127:117-30.

33. Berthoz A, Pozzo T. Intermittent head stabilization during postural and locomotor tasks in humans. Gait Posture 1988;189-98.

34. Prince F, Winter DA, Stergiou P, Walt SE. Anticipatory control of upper body balance during human locomotion. Gait Posture. 1994;2:19-25.

35. Winter DA, McFayden BJ, Dickey JP. Adaptability of the CNS in human walking. Advances Psychology 1991;78:127-44.

36. Hausdorff JM. Gait variability: methods, modeling and meaning. J Neuroeng Rehabil 2005;2:19.

37. Barak Y, Wagenaar RC, Holt KG. Gait characteristics of elderly people with a history of falls: a dynamic approach. Phys Ther 2006;86:1501-10.

38. Perlmutter E, Gregory PC. Rehabilitation treatment options for a patient with paraneoplastic cerebellar degeneration. Am J Phys Med Rehabil 2003;82:158-62.

39. Gibson-Horn C. Balance-based torso-weighting in a patient with ataxia and multiple sclerosis: a case report. J Neurol Phys Ther 2008;32:139-46.

40. Widener GL, Allen DD, Gibson-Horn C. Randomized clinical trial of balance-based torso weighting for improving upright mobility in people with multiple sclerosis. Neurorehabil Neural Repair 2009;23:784-91.

Conflicts of interest.-The authors certify that there is no conflict of interest with any financial organization regarding the material discussed in the manuscript. Congresses.-Data from the present study were presented at the 2014 International Conference on Neurorehabilitation (ICNR), which was held on June 24th-26th 2014 , in Aalborg, Denmark.

Article first published online: June 19, 2017. - Manuscript accepted: June 1, 2017. - Manuscript revised: April 21, 2017. - Manuscript received: September 7,2016.

For supplementary materials, please see the online version of this article. 


\section{COPYRIGHT $^{(} 2017$ EDIZIONI MINERVA MEDICA}

SERRAO

DYNAMIC MOVEMENT ORTHOSES IN ATAXIC PATIENTS

\section{SUPPLEMENTARY MATERIALS}

SuPPlementARY TABLE I.-Power analysis results.

\begin{tabular}{lc}
\hline \multicolumn{1}{c}{ Variables } & Power, 1- $\beta$ \\
\hline Stance phase duration & $59.11 \%$ \\
Double support duration & $84.49 \%$ \\
Knee ROM & $72.21 \%$ \\
Pelvis tilt ROM & $79.56 \%$ \\
Pelvis obliquity ROM & $58.23 \%$ \\
Anterior-posterior trunk ROM & $68.58 \%$ \\
CV of swing phase duration & $51.24 \%$ \\
\hline ROM: range of motion; CV: coefficient of variation. \\
\hline
\end{tabular}

